\title{
Low CFB expression is independently associated with poor overall and disease-free survival in patients with lung adenocarcinoma
}

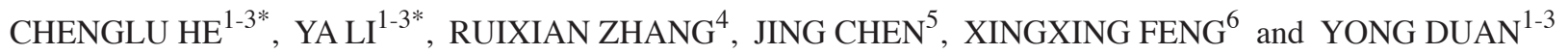 \\ ${ }^{1}$ Yunnan Key Laboratory of Laboratory Medicine; ${ }^{2}$ Yunnan Institute of Laboratory Diagnosis; \\ ${ }^{3}$ Department of Clinical Laboratory, The First Affiliated Hospital of Kunming Medical University, Kunming, Yunnan 650032; \\ ${ }^{4}$ Department of Environment-Related Health, Yunnan Center for Disease Control and Prevention, Kunming, Yunnan 650034; \\ ${ }^{5}$ Health Management Center, The First Affiliated Hospital of Kunming Medical University, Kunming, Yunnan 650032; \\ ${ }^{6}$ Department of Clinical Laboratory, Kunming Children's Hospital, Kunming, Yunnan 650228, P.R. China
}

Received August 21, 2020; Accepted March 16, 2021

DOI: $10.3892 / \mathrm{ol} .2021 .12739$

\begin{abstract}
Complement factor B (CFB) serves a pivotal role in the alternative signaling pathway of the complement system and exerts a key role in the labelling of target particles, resulting from effective clearance of the target. The present study aimed to investigate the association between low expression levels of CFB and the clinical features and survival status of patients with lung adenocarcinoma (LUAD). Patient data were based on RNA-sequencing and clinical data from The Cancer Genome Atlas database. All patients were divided into two groups based on the median expression of CFB. Kaplan-Meier curve and univariate Cox regression analyses were used to investigate the association between CFB and survival status. Gene set enrichment analysis was used to examine the effects of CFB expression on signaling pathway impairment. Furthermore, reverse transcription-quantitative PCR (RT-qPCR) and western blotting were used to verify the relative expression levels of CFB in LUAD tissues. The data revealed that residual tumor classification, Karnofsky performance score and cancer stage were associated with overall survival, and that Karnofsky performance score and stage were associated with disease-free survival. The results demonstrated that high expression levels of CFB were associated with increased patient overall and disease-free survival according to both continuous and categorical models. The results of multivariate analysis identified that high expression levels of CFB were associated with increased overall and disease-free survival according to both the continuous
\end{abstract}

Correspondence to: Dr Yong Duan, Department of Clinical Laboratory, The First Affiliated Hospital of Kunming Medical University, 295 Xichang Road, Kunming, Yunnan 650032, P.R. China E-mail: duanyong7@139.com

*Contributed equally

Key words: complement factor B, lung adenocarcinoma, bioinformatics, overall survival, disease-free survival model [hazard ratio (HR), 0.48; 95\% confidence interval (95\% CI), 0.25-0.93; $\mathrm{P}=0.029$ for overall survival; $\mathrm{HR}, 0.29$; 95\% CI, 0.15-0.59; $\mathrm{P}=0.001$ for disease-free survival] and the categorical model ( $\mathrm{HR}, 0.46 ; 95 \% \mathrm{CI}, 0.22-0.93 ; \mathrm{P}=0.031$ for overall survival; HR, 0.25; 95\% CI, 0.12-0.55; $\mathrm{P}=0.001$ for disease-free survival) after adjusting for corresponding covariates (residual tumour classification, Karnofsky performance score and stage). Furthermore, the results of both RT-qPCR and western blotting indicated that the relative mRNA and protein expression levels of CFB in lung tumor tissues were downregulated compared with those in adjacent non-tumor tissues. Collectively, the present results suggested that CFB expression was an independent predictor of overall and disease-free survival in patients with LUAD.

\section{Introduction}

Lung cancer is the leading cause of cancer-associated death among men, with a $24 \%$ mortality rate, and women, with a $23 \%$ mortality rate, in the United States (1). Based on its histology, lung adenocarcinoma (LUAD) is classified into two main forms: Non-small cell lung carcinoma (NSCLC) and small cell lung carcinoma, which comprise $\sim 85$ and $\sim 15 \%$ of all cases, respectively (2). NSCLC is further divided into three subtypes: Squamous-cell carcinoma, adenocarcinoma and large cell carcinoma. LUAD is the most common type of lung cancer, representing $\sim 40 \%$ of all lung cancer types (3). LUAD may occur due to mutations in a variety of genes, including EGFR, ALK receptor tyrosine kinase, KRAS, ROS proto-oncogene 1 , receptor tyrosine kinase, BRAF, erb-b2 receptor tyrosine kinase 2, MET proto-oncogene, receptor tyrosine kinase and ret proto-oncogene (4). At present, drugs targeting mutant genes, such as EGFR (5) and KRAS (6), associated with LUAD have been developed. However, LUAD is still one of the most aggressive and lethal tumor types, with a 5-year survival rate $<5 \%$ (7). Therefore, it is important to identify novel target genes to improve the prognosis of patients with LUAD.

Complement factor B (CFB), localized to the major histocompatibility complex class III region on chromosome 6 , serves a pivotal role in the alternative pathway of the complement 
system (8) and exerts a key role in labelling target particles, resulting from the effective clearance of the target (9). CFB is cleaved into two fragments, non-catalytic chain $\mathrm{Ba}$ and the catalytic subunit $\mathrm{Bb}$, by complement factor $\mathrm{D}$; the generated component $3 \mathrm{~b}(\mathrm{C} 3 \mathrm{~b})$ binds $\mathrm{Bb}$ and properdin, resulting in the formation of the $\mathrm{C} 3$ convertase $(\mathrm{C} 3 \mathrm{bBb})$ during the activation process of the alternative pathway (10). By binding of additional $\mathrm{C} 3 \mathrm{~b}$ to the alternative pathway, $\mathrm{C} 3 \mathrm{bBb}$ renders it able to cleave $\mathrm{C} 5$, and induces the amplification loop of the alternative pathway $(8,10)$. It has been reported that low expression levels of CFB are associated with decreased expression levels of $\mathrm{C} 3 \mathrm{bBb}$ and facilitate the degradation of the membrane attack complex (MAC), which leads to the inhibition of the alternative pathway of complement activation, thereby reducing the activation efficiency of the complement system in the whole body (8). During the regulation of the complex tumor microenvironment, complement proteins serve a dual role in the tumor microenvironment, which will eventually affect tumor progression (9). The dysregulation of complement activation pathways serves an important role in tumor progression (10-12).

A previous study reported that $\mathrm{CFB}$ is a candidate biomarker for pancreatic cancer diagnosis (13). Furthermore, it has been revealed that CFB expression is upregulated in patients with preeclampsia compared with in those with normotensive pregnancies (14). CFB has also been reported to serve a central role in mediating ultraviolet-induced immunosuppression (15). Previous data have indicated that CFB mRNA expression in the colonic mucosa is upregulated in the lower parts of the crypts compared with that observed on the luminal surface (16). Furthermore, a previous study has identified a strong association of CFB with molecular subtypes of breast cancer, including the luminal-A (LA), luminal-B, triple-negative and HER2-positive subtypes (17).

As a key immunomodulatory factor in the progression of lung cancer, complement activation induces escape from immunosurveillance via the $\mathrm{C} 3 / \mathrm{C} 5$-dependent signaling pathway, which is activated by classical signaling pathways (10), thereby affecting the occurrence and development of lung cancer (18). Therefore, the present study aimed to determine the role of CFB in LUAD using data from The Cancer Genome Atlas (TCGA). The present analysis provided further evidence regarding the use of CFB as a potential biomarker for LUAD, and CFB may become an attractive candidate biomarker and potential prognostic target for LUAD.

\section{Materials and methods}

Data collection. RNA-sequencing (seq) expression (combining level-3 data from Illumina GA and Hi-Seq platforms) and clinical data for patients with LUAD were downloaded from TCGA data portal (http://cancergenome.nih.gov/). The TCGA-LUAD dataset contained data on 514 patients with LUAD for whom detailed clinical information was available. RNA-seq by Expectation Maximization expression values were used for statistical analysis.

Clinical feature analysis. LUAD samples were divided into two groups based on the median CFB expression value of 3.64. All statistical analyses were performed using R statistical software (version 3.4.1; https://www.r-project.org/). The means of the continuous variables in these two groups (high-CFB group and low-CFB group) were compared using an independent sample t-test, and the prevalence of categorical variables was compared using the $\chi^{2}$ test. Due to the small sample size of the groups for some variables, the comparisons were performed using Pearson's $\chi^{2}$ test with Yates' continuity correction and Fisher's exact test. Violin plots were used to visualize expression level differences for discrete variables, such as sex, smoking history, residual tumor and stage. Univariate logistic regression was used to investigate the association between CFB expression (categorical) and clinical features.

Survival analysis. Differences in overall survival and disease-free survival between the high-CFB group and the low-CFB group were compared using Kaplan-Meier curves, with P-values calculated via the log-rank test using the 'survival' package (https://cran.r-project.org/web/packages/survival/index.html; version 3.2-7) in R. Univariate Cox regression analysis was used to estimate the independent effects of CFB expression and other clinical features, including age, sex, ethnicity, smoking history, residual tumor classification, Eastern Cooperative Oncology Group (ECOG) score (19), Karnofsky performance score (20) and TNM stage (21), on overall survival and disease-free survival. The independent effect of CFB expression on overall survival and disease-free survival was evaluated via multivariate Cox analysis with adjusted covariates. For the sub-group analysis, patients were divided into three or four groups according to the tercile or quartile expression of CFB.

Gene set enrichment analysis (GSEA). The JavaGSEA desktop application v3.0 was used to perform GSEA (22) of Kyoto Encyclopedia of Genes and Genomes (KEGG; https://www.genome.jp/kegg/) pathways for the following comparisons: High-CFB vs. control, low-CFB vs. control and high-CFB vs. low-CFB. Gene sets with $<10$ genes or $>500$ genes were excluded. The t-statistic mean of the genes was computed for each metabolic pathway using a permutation test with 1,000 replications. Up- and downregulated metabolic pathways were defined as having a normalized enrichment score (NES) $>0$ or $<0$ for patients compared with controls, respectively. Enrichment analysis of Gene Ontology (https://www.ebi.ac.uk/QuickGO/) biological processes and hallmark pathways was also conducted. An absolute value of NES $>1$ and a false discovery rate-corrected P-value $\leq 0.05$ were considered significant.

Patients and tissue samples. Written informed consent was obtained according to the guidelines of the Medical Ethics Committee of The First Affiliated Hospital of Kunming Medical University (Kunming, China). Between July and August 2020, a total of 3 patients who were pathologically diagnosed with NSCLC at The First Affiliated Hospital of Kunming Medical University (Kunming, China), including 2 male patients and 1 female patient (maximum age, 64 years; minimum age, 55 years; median age, 65 years), were recruited in the present study, and tumor types were confirmed by $\geq 2$ experienced pathologists. The six paired samples collected were used to identify the expression levels of CFB in lung tumor tissues. The enrolled patients met the following criteria: 
i) No patients diagnosed as LUAD by pathology had received chemotherapy or radiotherapy; ii) the adjacent non-tumor lung tissues were collected $\geq 5 \mathrm{~cm}$ away from carcinoma tissues; iii) the lung tissue samples collected during surgery were frozen in liquid nitrogen $\left(-196^{\circ} \mathrm{C}\right)$ within $30 \mathrm{~min}$; and iv) tumor tissue samples contained $\geq 80 \%$ typical tumor cellularity, while the matched adjacent non-tumor lung tissues samples contained no cancer cells.

Reverse transcription-quantitative PCR (RT-qPCR). Total RNA from tissues was isolated using TRIzol ${ }^{\circledR}$ reagent (Invitrogen; Thermo Fisher Scientific, Inc.) according to the manufacturer's protocol. cDNA was synthesized using 5X All-In-One MasterMix (cat. no. G492; Applied Biological Materials, Inc.). The mixture was incubated at $25^{\circ} \mathrm{C}$ for $10 \mathrm{~min}$ and then at $42^{\circ} \mathrm{C}$ for $15 \mathrm{~min}$. SYBR-Green Master mix (cat. no. MasterMix-R; Applied Biological Materials, Inc.) was used to perform qPCR on a 7300 Real-Time PCR system (Applied Biosystems; Thermo Fisher Scientific, Inc.). Initially, the enzyme in the mixture was activated at $95^{\circ} \mathrm{C}$ for $10 \mathrm{~min}$ for 1 cycle, then the mixture was denaturalized at $95^{\circ} \mathrm{C}$ for $15 \mathrm{sec}$ and annealed/extended at $60^{\circ} \mathrm{C}$ for $60 \mathrm{sec}$ for 40 cycles. The relative mRNA expression in different samples was calculated using the $2^{-\Delta \Delta \mathrm{Cq}}$ normalization method (23). The following primers were used: CFB forward, 5'-AATCAAGGTCAGCGTAGGAGG-3' and reverse, 5'-GGGAGACAAATGGGCCTGATA-3'. GAPDH was chosen as an internal control using the following primers: GAPDH forward, 5'-GCATCCTGGGCTACACTGAG-3' and reverse, 5'-AAGTGGTCGTTGAGGGCAAT-3'.

Western blotting. Total protein from tissues was extracted using RIPA lysis buffer (Beijing Solarbio Science \& Technology Co., Ltd.) containing protease inhibitor (EMD Millipore), and the protein concentration was determined using a BCA protein assay (Beijing Solarbio Science \& Technology Co.,Ltd.). A total 10-15 $\mu \mathrm{g}$ protein/lane was separated via 8-15\% SDS-PAGE and transferred to PVDF membranes, which were incubated with specific primary antibodies overnight at $4^{\circ} \mathrm{C}$ after blocking with $5 \%$ non-fat milk at room temperature for $1 \mathrm{~h}$. Primary antibodies used in the present study included anti-CFB (Abcam; cat. no. ab133765; 1:5,000) and anti-GAPDH (Cell Signaling Technology, Inc.; cat. no. 8884; 1:1,000), which were diluted in $1 \mathrm{X}$ TBS-Tween (TBST; containing 0.1\% Tween-20). The membranes were washed with $1 \mathrm{X}$ TBST and incubated at room temperature for $1 \mathrm{~h}$ with HRP-conjugated anti-rabbit IgG secondary antibodies (Cell Signaling Technology, Inc.; cat. no. 7074; 1:10,000) diluted in 1X TBST. Finally, Protein bands were visualized using a High-Sig ECL Western Blotting kit (Tanon Science and Technology Co., Ltd.), and the band intensity value was subsequently measured using ImageJ software (v1.8.0; National Institutes of Health) with GAPDH serving as an internal control.

Statistical analysis. All the experiments, including RT-qPCR and western blotting, were repeated three times. All quantitative data were presented as the mean \pm standard error and analyzed using SPSS software (version 22.0; IBM Corp) and GraphPad Prism 8 (GraphPad Software, Inc.). A paired-sample t-test was performed to compare the relative mRNA expression levels of the CFB gene between tumor and matched adjacent non-tumor tissues. A Wilcoxon rank sum test was used for comparisons of protein levels in two independent groups. $\mathrm{P}<0.05$ was considered to indicate a statistically significant difference.

\section{Results}

Patient characteristics. The characteristics of patients with LUAD are presented in Table I. There was no significant difference in age, sex, ethnicity, residual tumor classification, ECOG score or Karnofsky performance score between the low-CFB and high-CFB groups. There was a higher percentage of former smokers and a lower percentage of non-smokers and current smokers in the low-CFB group compared with in the high-CFB group ( $\mathrm{P}=0.021)$. Furthermore, patients in the low-CFB group had a higher stage (stage III and stage IV) compared with patients in the high-CFB group $(\mathrm{P}=0.010)$. Additionally, the violin plot indicated a negative association trend between $\mathrm{CFB}$ expression and stage (Fig. 1G). Furthermore, other clinical features showed no or an inconsistent association with CFB expression (Fig. 1A-F).

CFB expression and clinical features. The associations between CFB expression and clinical features are shown in Table II. There were more former smokers in the low-CFB group compared with in the high-CFB group $(\mathrm{P}=0.026$ for former smokers vs. non-smokers). Furthermore, there were more stage III patients in the low-CFB group compared with in the high-CFB group ( $\mathrm{P}=0.002$ for stage III vs. stage I). Other clinical features (age, sex, ethnicity, residual tumor classification, ECOG score and Karnofsky performance score) were not observed to be associated with CFB expression.

CFB expression, clinical features and patient survival. Data on CFB expression, clinical features and patient survival are presented in Tables III and IV. High CFB expression was significantly associated with overall survival and disease-free survival according to both the continuous $(\mathrm{P}=0.045$ for overall survival; $\mathrm{P}=0.006$ for disease-free survival) and categorical model $(\mathrm{P}=0.005$ for overall survival; $\mathrm{P}=0.002$ for disease-free survival). The Kaplan-Meier curves suggested that the low-CFB group had a significantly decreased overall survival (Fig. 1H) and disease-free survival (Figs. 1I and S1) compared with the high-CFB group. In the sub-group analysis of different smokers, there was no significant association between CFB expression and overall survival or disease-free survival (Fig. S2). Furthermore, the residual tumor classification was significantly associated with overall survival $(\mathrm{P}<0.001$ for R1 vs. R0). The Karnofsky performance score was significantly associated with overall survival $(\mathrm{P}=0.031)$ and disease-free survival $(\mathrm{P}=0.013)$. Additionally, high stage was significantly associated with overall survival $(\mathrm{P}<0.001$ for stage II vs. stage I; $\mathrm{P}<0.001$ for stage III vs. stage I; $\mathrm{P}<0.001$ for stage IV vs. stage I) and disease-free survival $(\mathrm{P}<0.001$ for stage II vs. stage I; $\mathrm{P}<0.001$ for stage III vs. stage I; $\mathrm{P}=0.034$ for stage IV vs. stage I). These results indicated that the residual tumor classification, Karnofsky performance score and cancer stage were associated with overall survival, and that Karnofsky performance score and stage were associated with disease-free survival. 
Table I. Descriptive statistics stratified by CFB expression.

\begin{tabular}{|c|c|c|c|}
\hline Variable $^{\mathrm{a}}$ & CFB $<3.64(n=257)$ & CFB $\geq 3.64(n=256)$ & P-value \\
\hline Mean age $\pm \mathrm{SD}$, years & $65.44 \pm 10.25$ & $65.28 \pm 9.62$ & 0.855 \\
\hline Sex, n $(\%)$ & & & 0.143 \\
\hline Male & $128(49.81)$ & $110(42.97)$ & \\
\hline Female & $129(50.19)$ & $146(57.03)$ & \\
\hline Ethnicity, n (\%) & & & 0.568 \\
\hline Asian & $2(0.91)$ & $5(2.18)$ & \\
\hline White & $191(87.21)$ & $196(85.59)$ & \\
\hline Black or African American & $25(11.42)$ & $28(12.23)$ & \\
\hline American Indian or Alaska Native & $1(0.46)$ & $0(0.00)$ & \\
\hline Smoking history, n (\%) & & & 0.021 \\
\hline Non-smoker & $30(12.15)$ & $45(17.86)$ & \\
\hline Former smoker & $166(67.21)$ & $139(55.16)$ & \\
\hline Current smoker & $51(20.65)$ & $68(26.98)$ & \\
\hline Residual tumour classification, n (\%) & & & 0.716 \\
\hline R0 & $172(96.09)$ & $172(94.51)$ & \\
\hline $\mathrm{R} 1$ & $5(2.79)$ & $8(4.40)$ & \\
\hline $\mathrm{R} 2$ & $2(1.12)$ & $2(1.10)$ & \\
\hline ECOG score (mean \pm SD) & $0.61 \pm 0.62$ & $0.76 \pm 0.75$ & 0.120 \\
\hline Karnofsky performance score (mean \pm SD) & $86.46 \pm 20.37$ & $84.40 \pm 18.53$ & 0.602 \\
\hline Stage, n (\%) & & & 0.010 \\
\hline Stage I & $120(48.00)$ & $155(60.78)$ & \\
\hline Stage II & $62(24.80)$ & $59(23.14)$ & \\
\hline Stage III & $53(21.20)$ & $31(12.16)$ & \\
\hline Stage IV & $15(6.00)$ & $10(3.92)$ & \\
\hline
\end{tabular}

${ }^{a}$ The continuous variables are presented as the mean \pm SD, and the means for these variables in the two groups were compared using unpaired Student's t-test. The categorical variables are presented as numbers (percentages of cases), and the prevalence of these variables was compared using Pearson's $\chi^{2}$ test. Due to the small sample size of the groups for some variables, the comparisons were performed using Pearson's $\chi^{2}$ test with Yates' continuity correction and Fisher's exact test. Some data for ethnicity, smoking history, residual tumour classification, ECOG score, Karnofsky performance score and stage were missing, so the numbers are less than the total number of samples. CFB, complement factor B; ECOG, Eastern Cooperative Oncology Group.

The results of the multivariate Cox regression analysis demonstrated that high CFB expression was associated with increased overall survival time (Table V) and disease-free survival time (Table VI), according to both the continuous model [hazard ratio (HR), 0.48; $95 \%$ confidence interval (95\% CI), 0.25-0.93; $\mathrm{P}=0.029$ for overall survival; HR, 0.29; 95\% CI, 0.15-0.59; $\mathrm{P}=0.001$ for disease-free survival] and the categorical model (HR, 0.46; 95\% CI, 0.22-0.93; $\mathrm{P}=0.031$ for overall survival; HR, $0.25 ; 95 \% \mathrm{CI}, 0.12-0.55$; $\mathrm{P}=0.001$ for disease-free survival) after adjusting for corresponding covariates (residual tumour classification, Karnofsky performance score and stage).

CFB expression and pathway impairment. Significantly enriched KEGG pathways of the three groups are presented in Fig. 2. There were four deregulated KEGG pathways for the high-CFB vs. control, including 'steroid biosynthesis', 'nicotinate and nicotinamide metabolism', 'glutamatergic synapse' and 'autophagy', whereas only 'nicotinate and nicotinamide metabolism' was upregulated in the low-CFB vs. control group.
Furthermore, there were 17 downregulated pathways in the high-CFB vs. low-CFB group. These findings indicated that there was a marked difference in the pathway deregulation of individuals with different CFB expression states. Enrichment analysis of Gene Ontology biological processes (Table SI) and hallmark pathways (Table SII) was also conducted. The results demonstrated that CFB was associated with multiple signaling pathways related to cell proliferation and tumorigenesis, such as 'positive regulation of $\mathrm{G} 2 / \mathrm{M}$ transition of mitotic cell cycle', 'TNFA signaling via NF- $\mathrm{B}$ ' and 'Wnt/ $\beta$-catenin signaling'.

$C F B$ expression in human lung tissues. It was identified that both the relative mRNA and protein expression levels of CFB were downregulated in tumor tissues compared with in matched adjacent non-tumor tissues $(\mathrm{P}<0.05$; Fig. 3$)$.

\section{Discussion}

Increasing attention has been paid to precise individualized medicine in cancer treatment, which is facilitated by the explo- 


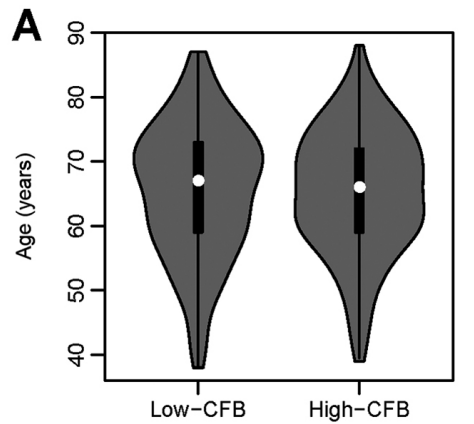

CFB (categorical)
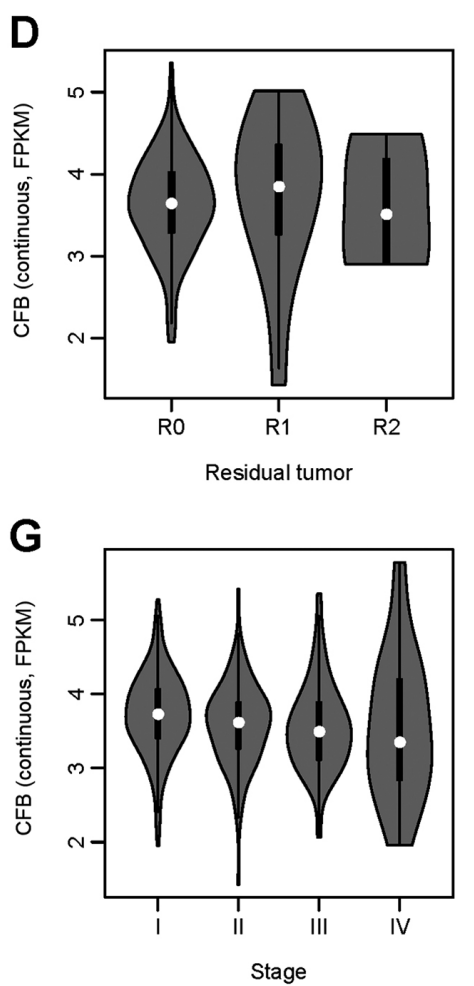

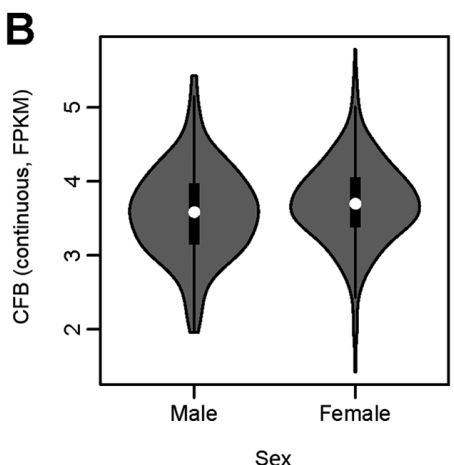

E
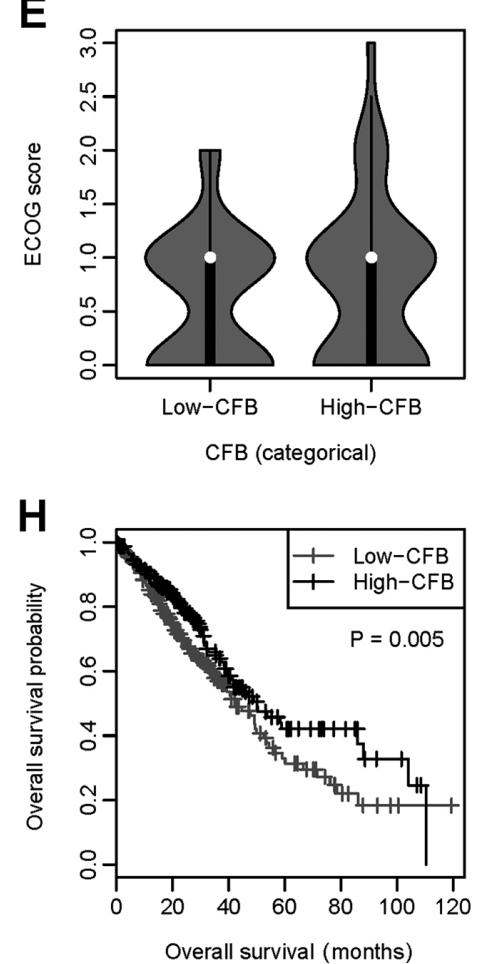
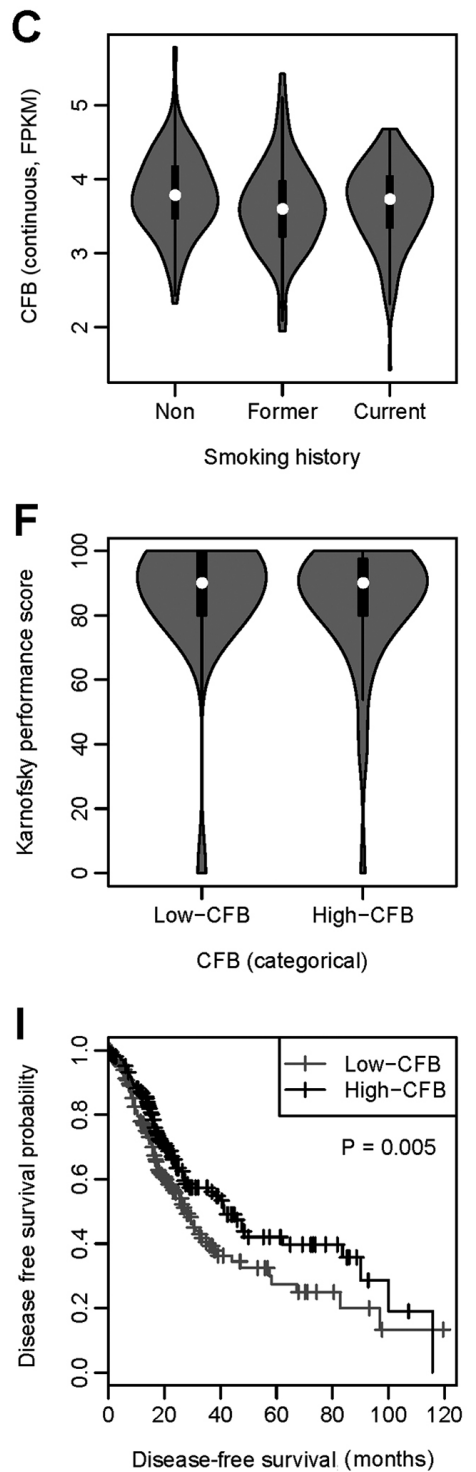

Figure 1. CFB expression and its association with clinical features. Association between CFB expression and (A) age, (B) sex, (C) smoking history, (D) residual tumor classification, (E) ECOG score, (F) Karnofsky performance score, (G) stage, (H) overall survival and (I) disease-free survival. Low-CFB, CFB expression <3.64; high-CFB, CFB expression $\geq 3.64$. CFB, complement factor B; ECOG, Eastern Cooperative Oncology Group.

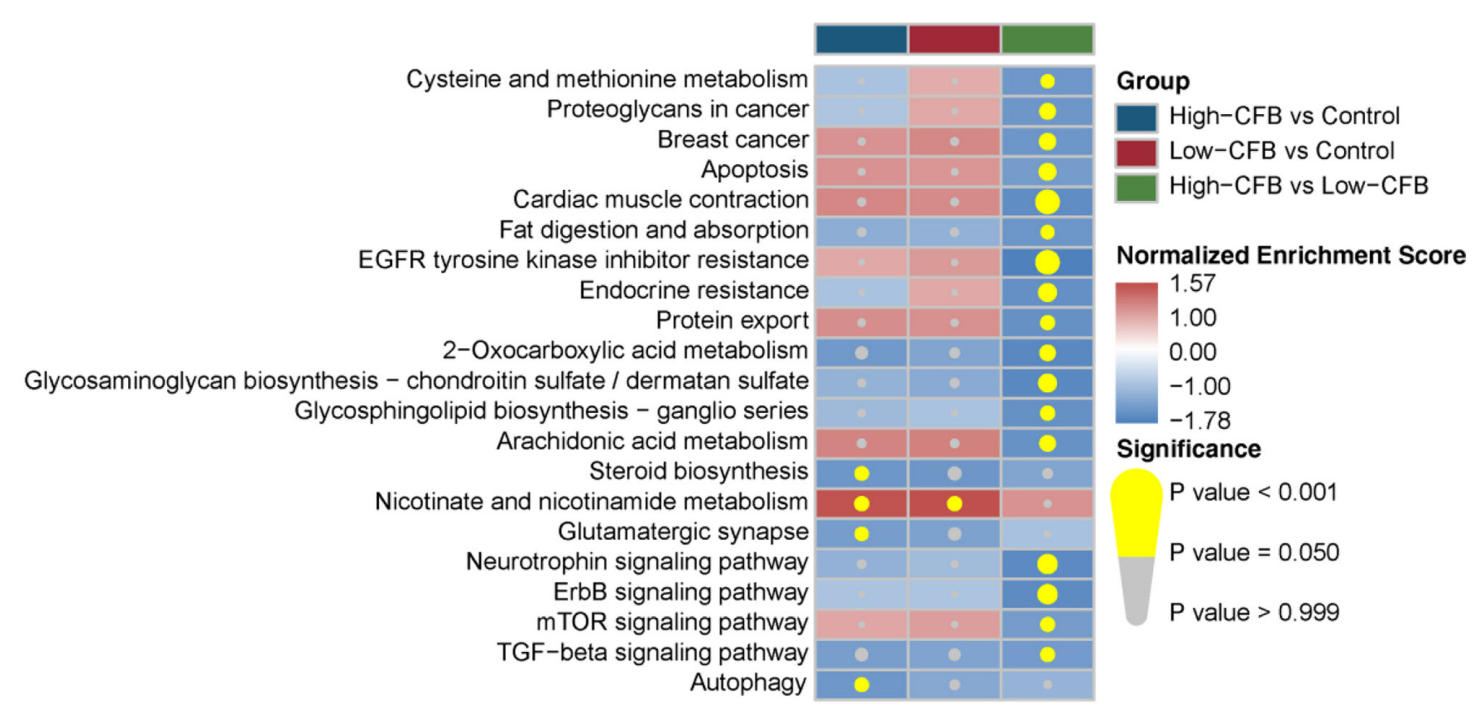

Figure 2. Gene set enrichment analysis results of Kyoto Encyclopedia of Genes and Genomes pathways in three groups. A red box indicates that the pathway is upregulated and a blue box indicates that the pathway is downregulated. A yellow circle indicates that the pathway is significantly enriched. CFB, complement factor B. 
Table II. Univariate logistic regression analysis of clinical features and CFB expression.

\begin{tabular}{|c|c|c|c|}
\hline Variable & $\mathrm{N} 1 / \mathrm{N} 2^{\mathrm{a}}$ & OR $(95 \% \mathrm{CI})$ & P-value \\
\hline Age (years) & & $1.00(0.98-1.02)$ & 0.854 \\
\hline \multicolumn{4}{|l|}{ Sex } \\
\hline Male & $128 / 110$ & Reference & \\
\hline Female & $129 / 146$ & $1.32(0.93-1.86)$ & 0.121 \\
\hline \multicolumn{4}{|l|}{ Ethnicity } \\
\hline Asian & $2 / 5$ & Reference & \\
\hline White & 191/196 & - & - \\
\hline Black or African American & $25 / 28$ & - & - \\
\hline American Indian or Alaska Native & $1 / 0$ & - & - \\
\hline \multicolumn{4}{|l|}{ Smoking history } \\
\hline Non-smoker & $30 / 45$ & Reference & \\
\hline Former smoker & $166 / 139$ & $0.56(0.33-0.93)$ & 0.026 \\
\hline Current smoker & $51 / 68$ & $0.89(0.49-1.60)$ & 0.694 \\
\hline \multicolumn{4}{|l|}{ Residual tumour classification } \\
\hline R0 & $172 / 172$ & Reference & \\
\hline $\mathrm{R} 1$ & $5 / 8$ & $1.60(0.51-4.99)$ & 0.418 \\
\hline R2 & $2 / 2$ & - & - \\
\hline ECOG score & & $1.36(0.92-2.01)$ & 0.129 \\
\hline Karnofsky performance score & & $0.99(0.97-1.02)$ & 0.599 \\
\hline \multicolumn{4}{|l|}{ Stage } \\
\hline Stage I & $120 / 155$ & Reference & \\
\hline Stage II & $62 / 59$ & $0.74(0.48-1.13)$ & 0.163 \\
\hline Stage III & $53 / 31$ & $0.45(0.27-0.75)$ & 0.002 \\
\hline Stage IV & $15 / 10$ & $0.52(0.22-1.19)$ & 0.120 \\
\hline
\end{tabular}

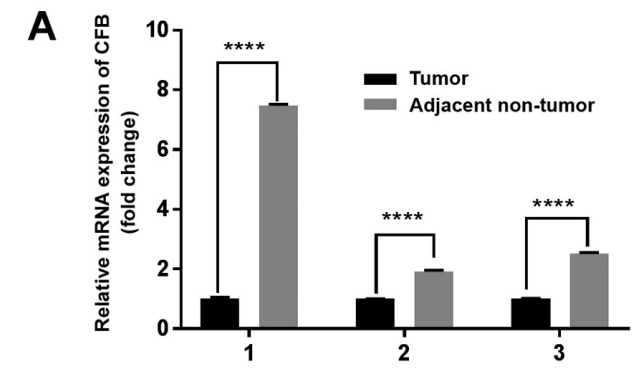

B

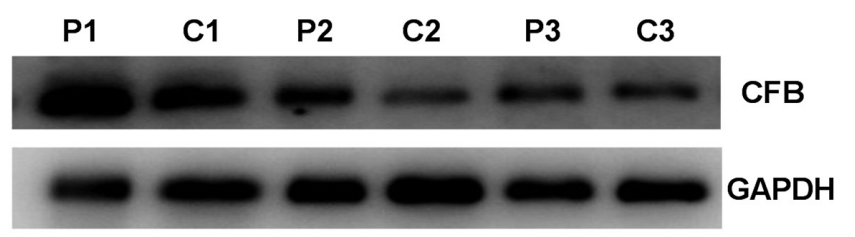

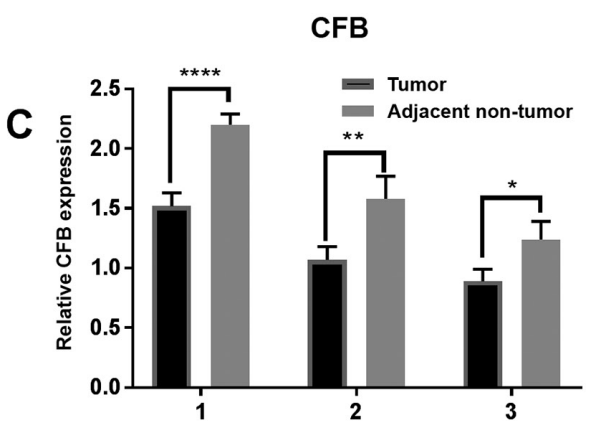

Figure 3. Identification of downregulated CFB expression in 3 paired lung adenocarcinoma samples. (A) Relative mRNA expression levels of CFB were decreased in tumor tissue as detected by reverse transcription-quantitative PCR $(n=6)$. (B) Relative downregulated protein expression levels of CFB in lung adenocarcinoma samples as detected by western blotting $(n=6)$. (C) CFB protein expression was decreased in tumor tissues compared with in matched adjacent non-tumor tissues. Both the relative mRNA and protein expression levels of CFB in each patient's lung tumor and adjacent non-tumor tissue were normalized against GAPDH values as determined in three replicate experiments. ${ }^{*} \mathrm{P}<0.05 ;{ }^{* *} \mathrm{P}<0.01 ;{ }^{* * * *} \mathrm{P}<0.0001$. $\mathrm{C}$, lung adenocarcinoma tissue; $\mathrm{P}$, matched adjacent non-tumor tissue; CFB, complement factor B. 
Table III. Univariate Cox proportional hazards regression analysis of CFB expression, clinical features and overall survival.

\begin{tabular}{|c|c|c|c|c|}
\hline Variable & $\mathrm{N}$ & Median survival time (Q1-Q3), months & $\mathrm{HR}(95 \% \mathrm{CI})$ & P-value \\
\hline CFB (continuous) & 504 & & $0.77(0.60-0.99)$ & 0.045 \\
\hline \multicolumn{5}{|l|}{ CFB (categorical) } \\
\hline CFB $<3.64$ & 250 & $20.60(11.55-35.12)$ & Reference & \\
\hline $\mathrm{CFB} \geq 3.64$ & 254 & 21.95 (14.67-38.68) & $0.66(0.49-0.88)$ & 0.005 \\
\hline Age (years) & 494 & & $1.01(0.99-1.02)$ & 0.324 \\
\hline \multicolumn{5}{|l|}{ Sex } \\
\hline Male & 235 & $20.66(10.58-36.13)$ & Reference & \\
\hline Female & 269 & $21.62(14.72-37.12)$ & $0.96(0.72-1.29)$ & 0.808 \\
\hline \multicolumn{5}{|l|}{ Ethnicity } \\
\hline Asian & 7 & $18.66(7.66-24.21)$ & Reference & \\
\hline White & 387 & $20.83(13.63-35.35)$ & NA & NA \\
\hline Black or African American & 53 & $22.01(16.85-37.29)$ & NA & NA \\
\hline American Indian or Alaska Native & 1 & $15.34(15.34-15.34)$ & NA & NA \\
\hline \multicolumn{5}{|l|}{ Smoking history } \\
\hline Non-smoker & 72 & $23.66(13.84-36.09)$ & Reference & \\
\hline Former smoker & 300 & $20.02(13.44-35.26)$ & $0.92(0.60-1.41)$ & 0.695 \\
\hline Current smoker & 118 & $22.08(14.60-36.73)$ & $0.84(0.51-1.37)$ & 0.476 \\
\hline \multicolumn{5}{|l|}{ Residual tumour classification } \\
\hline R0 & 336 & $23.14(14.59-39.77)$ & Reference & \\
\hline $\mathrm{R} 1$ & 13 & $21.98(9.56-29.99)$ & $3.33(1.73-6.40)$ & $<0.001$ \\
\hline $\mathrm{R} 2$ & 3 & $8.02(6.00-11.52)$ & NA & NA \\
\hline ECOG score & 213 & & $1.33(0.97-1.82)$ & 0.076 \\
\hline Karnofsky performance score & 97 & & $0.99(0.97-1.00)$ & 0.031 \\
\hline \multicolumn{5}{|l|}{ Stage } \\
\hline Stage I & 271 & $22.80(15.64-41.85)$ & Reference & \\
\hline Stage II & 119 & $22.24(11.11-32.75)$ & $2.53(1.76-3.65)$ & $<0.001$ \\
\hline Stage III & 81 & $15.37(8.80-28.88)$ & $3.61(2.46-5.29)$ & $<0.001$ \\
\hline Stage IV & 25 & $21.55(15.01-31.11)$ & $4.07(2.33-7.11)$ & $<0.001$ \\
\hline
\end{tabular}

Data are presented as the median (Q1-Q3 quantiles) survival time, and the regression results are presented as HR (95\% CI) and P-values. Some data for ethnicity, smoking history, residual tumour classification, ECOG score, Karnofsky performance score and stage were missing, so the numbers are less than the total number of samples. N, number of samples. 95\% CI, 95\% confidence interval; CFB, complement factor B; ECOG, Eastern Cooperative Oncology Group; HR, hazard ratio; NA, not available.

ration and identification of biomarkers (24). The treatment of LUAD is associated with complex factors and comprises multiple targeted therapies. Therefore, a greater understanding of molecular biomarkers will help to improve the diagnosis and prognosis of human LUAD.

CFB is a key soluble component in the alternative pathway (8). CFB is cleaved by complement factor D into fragments $\mathrm{Ba}$ and $\mathrm{Bb}$. The complement factor $\mathrm{D}$ and activated component $\mathrm{Bb}$ are serine proteinases. Furthermore, $\mathrm{Bb}$ remains attached to $\mathrm{C} 3 \mathrm{~b}$ and forms the alternative pathway convertase, $\mathrm{C} 3 \mathrm{bBb}$, which is a key enzyme involved in the activation of the alternative pathway (10). It has been demonstrated that low CFB expression is associated with decreased $\mathrm{C} 3 \mathrm{bBb}$ expression and accelerates the degradation of the MAC, which leads to inhibition of the alternative pathway of complement activation, thereby significantly reducing the activation efficiency of the complement system in the whole body (8). During the regulation of the complex tumor microenvironment, complement proteins serve a dual role in the tumor microenvironment, which will eventually affect tumor progression $(8,9)$. The present study demonstrated that there was no difference in age, sex, ethnicity, residual tumor classification, ECOG score or Karnofsky performance score between the low-CFB and high-CFB groups. Furthermore, there were more former smokers and stage III patients in the high-CFB group compared with in the low-CFB group. Notably, in both univariate and multivariate analysis, high $\mathrm{CFB}$ expression was associated with overall survival and disease-free survival, according to both the continuous and categorical models.

CFB is a serum protein that is not only produced by the liver (25-27) but can also be synthesized by the choroid, retinal pigment epithelial cells and neural retina (28). Furthermore, the CFB protein has been found in ocular drusen and Bruch's membrane (26), and was observed to be potently upregulated 
Table IV. Univariate Cox proportional hazards regression analysis of CFB expression, clinical features and disease-free survival.

\begin{tabular}{|c|c|c|c|c|}
\hline Variable & $\mathrm{N}$ & Median survival time (Q1-Q3), months & $\mathrm{HR}(95 \% \mathrm{CI})$ & P-value \\
\hline CFB (continuous) & 428 & & $0.70(0.55-0.90)$ & 0.006 \\
\hline \multicolumn{5}{|l|}{ CFB (categorical) } \\
\hline CFB $<3.64$ FPKM & 207 & $16.79(8.15-27.76)$ & Reference & \\
\hline $\mathrm{CFB} \geq 3.64 \mathrm{FPKM}$ & 221 & $19.74(13.76-31.18)$ & $0.62(0.47-0.84)$ & 0.002 \\
\hline Age (years) & 418 & & $1.01(0.99-1.02)$ & 0.317 \\
\hline \multicolumn{5}{|l|}{ Sex } \\
\hline Male & 193 & $17.61(8.54-29.01)$ & Reference & \\
\hline Female & 235 & $18.17(13.27-28.70)$ & $0.97(0.72-1.29)$ & 0.822 \\
\hline \multicolumn{5}{|l|}{ Ethnicity } \\
\hline Asian & 7 & $14.49(6.00-22.88)$ & Reference & \\
\hline White & 329 & $17.44(10.09-27.23)$ & $0.62(0.23-1.69)$ & 0.354 \\
\hline Black or African American & 48 & $20.16(15.66-33.78)$ & $0.45(0.15-1.35)$ & 0.156 \\
\hline American Indian or Alaska Native & 1 & $9.26(9.26-9.26)$ & NA & NA \\
\hline \multicolumn{5}{|l|}{ Smoking history } \\
\hline Non-smoker & 60 & $17.92(13.12-31.47)$ & Reference & \\
\hline Former smoker & 259 & $16.92(9.54-26.25)$ & $1.07(0.70-1.64)$ & 0.758 \\
\hline Current smoker & 97 & $20.70(13.40-30.98)$ & $0.74(0.45-1.24)$ & 0.253 \\
\hline \multicolumn{5}{|l|}{ Residual tumour classification } \\
\hline R0 & 282 & $18.99(12.41-33.02)$ & Reference & \\
\hline $\mathrm{R} 1$ & 11 & $14.72(5.18-18.84)$ & NA & NA \\
\hline ECOG score & 181 & & $1.03(0.73-1.45)$ & 0.880 \\
\hline Karnofsky performance score & 79 & & $0.98(0.97-1.00)$ & 0.013 \\
\hline \multicolumn{5}{|l|}{ Stage } \\
\hline Stage I & 246 & $19.22(13.63-31.82)$ & Reference & \\
\hline Stage II & 102 & $16.98(8.21-26.88)$ & $2.16(1.54-3.02)$ & $<0.001$ \\
\hline Stage III & 59 & $13.76(7.23-24.95)$ & $2.23(1.47-3.37)$ & $<0.001$ \\
\hline Stage IV & 14 & $19.88(14.02-25.67)$ & $2.22(1.06-4.62)$ & 0.034 \\
\hline
\end{tabular}

Data are presented as the median (Q1-Q3 quantiles) survival time, and the regression results are presented as HR (95\% CI) and P-values. Some data for ethnicity, smoking history, residual tumour classification, ECOG score, Karnofsky performance score and stage were missing, so the numbers are less than the total number of samples. N, number of samples. 95\% CI, 95\% confidence interval; CFB, complement factor B; ECOG, Eastern Cooperative Oncology Group; HR, hazard ratio; NA, not available.

in patients with ulcerative colitis and Crohn's disease (29). Previous data have demonstrated that CFB may be highly expressed in association with inflammatory bowel disease, in addition to having a possible key role in systemic complement activation (30). A previous study has reported that polymorphisms in CFB are associated with the risk of age-related macular degeneration (31). Furthermore, the study of CFB non-synonymous variants may improve the understanding of chronic hepatitis B etiology (31). The present study first used online public data analysis to demonstrate that low CFB expression was associated with decreased overall and disease-free survival in patients with LUAD. Subsequently, the prognostic value of CFB expression in lung tumor tissues was further investigated.

Recent studies have reported that CFB is associated with the prognosis of different types of cancer (32-34). A previous study has demonstrated that CFB is not only identified in all crypts in the colonic mucosa, but is also expressed in adenomas and carcinomas (16). The expression levels of CFB have been revealed to be increased in the plasma of patients with pancreatic cancer, for which CFB may be a novel biomarker (13). A previous analysis of CFB genetic alterations and mRNA expression in breast cancer has been performed using public TCGA invasive breast carcinoma sample data (32). CFB exhibits a strong association with molecular subtypes of breast cancer, particularly the LA subtype (17). Furthermore, $\mathrm{CFB}$ is a potential biomarker in pancreatic ductal adenocarcinoma (13) and pancreatic cancer (32) with diagnostic significance, and is also associated with a high likelihood of relapse-free survival (17). To the best of our knowledge, there has been no study addressing the prognostic significance of CFB in patients with LUAD. In the present study, multivariate Cox regression analysis demonstrated that high CFB expression was associated with increased overall and disease-free survival according to a continuous model after adjusting for corresponding covariates. Consistent with these results, both 
Table V. Multivariate Cox proportional hazards regression analysis of CFB expression and overall survival.

\begin{tabular}{lccc}
\hline Variable & $\mathrm{N}$ & Median survival time (Q1-Q3), months & HR (95\% CI) \\
\hline CFB (continuous) & 504 & & $0.48(0.25-0.93)$ \\
CFB (categorical) & & & 0.029 \\
CFB <3.64 FPKM & 250 & $20.60(11.55-35.12)$ & Reference \\
CFB $\geq 3.64$ FPKM & 254 & $21.95(14.67-38.68)$ & $0.46(0.22-0.93)$ \\
\hline
\end{tabular}

Data are presented as the median (Q1-Q3 quantiles) survival time, and the regression results are presented as HR (95\% CI) and adjusted P-values. $\mathrm{N}$, number of samples. P-values were adjusted for residual tumour classification, Karnofsky performance score and stage. 95\% CI, 95\% confidence interval; CFB, complement factor B; HR, hazard ratio.

Table VI. Multivariate Cox proportional hazards regression analysis of CFB expression and disease-free survival.

\begin{tabular}{lccc}
\hline Variable & $\mathrm{N}$ & Median survival time (Q1-Q3), months & HR (95\% CI) \\
\hline CFB (continuous) & 428 & & $0.29(0.15-0.59)$ \\
CFB (categorical) & & & 0.001 \\
CFB <3.64 FPKM & 207 & $16.79(8.15-27.76)$ & Reference \\
CFB $\geq 3.64$ FPKM & 221 & $19.74(13.76-31.18)$ & $0.25(0.12-0.55)$ \\
\hline
\end{tabular}

Data are presented as the median (Q1-Q3 quantiles) survival time, and the regression results are presented as HR (95\% CI) and adjusted P-values. N, number of samples. P-values were adjusted for Karnofsky performance score and stage. 95\% CI, 95\% confidence interval; $\mathrm{CFB}$, complement factor $\mathrm{B}$; HR, hazard ratio.

RT-qPCR and western blotting indicated that the relative expression levels of the CFB gene in lung tumor tissues were decreased compared with those in adjacent non-tumor tissues.

There are several limitations to the present study. First, the association between $\mathrm{CFB}$ and the occurrence and progression of LUAD, as well as the specific pathogenesis, remains unknown. The current results can to some extent explain an association between CFB and LUAD; however, prospective randomized controlled studies are required to confirm these promising results. Second, the current data concerning drug therapy and prognosis of LUAD are not widely available. Given the limitations of the present study, further large-sample and in-depth studies are required to confirm these results.

In conclusion, the present study demonstrated that $\mathrm{CFB}$ expression was an independent predictor of overall and disease-free survival in patients with LUAD. The current results may therefore provide helpful information for the early diagnosis and drug development of LUAD.

\section{Acknowledgements}

Not applicable

\section{Funding}

The present study was supported by grants from the National Natural Science Foundation of China (grant nos. 81460325 and 81760384), the Applied Basic Research in Yunnan Province (grant no. 2015FB040), and the Health Science and Technology Project in Yunnan Province (grant no. 2017NS030). The funders had no role in the study design, data collection and analysis, decision to publish or preparation of the manuscript.

\section{Availability of data and materials}

The datasets used and/or analyzed during the current study are available from the corresponding author on reasonable request.

\section{Authors' contributions}

$\mathrm{CH}$ and YL contributed to the design of the present study, developed the methodology, collected the bioinformatics data, performed the experiments, analyzed the results and wrote the manuscript. RZ and JC contributed to the collection of patient data and assisted with qPCR and western blotting experiments. $\mathrm{XF}$ assisted in experimental design, data analysis and manuscript editing. YD contributed to the design of the study, critically revised the manuscript and approved the final version to be published. CH, YL and YD confirmed the authenticity of all raw data. All authors have read and approved the final manuscript.

\section{Ethics approval and consent to participate}

The present study was approved by the Ethics Committee of the First Affiliated Hospital of Kunming Medical University (Kunming, China; approval no. 2020L42). Written informed consent was obtained from patients.

\section{Patient consent for publication}

Not applicable.

\section{Competing interests}

The authors declare that they have no competing interests. 


\section{References}

1. Siegel RL, Miller KD and Jemal A: Cancer statistics, 2019. CA Cancer J Clin 69: 7-34, 2019.

2. Jackman DM and Johnson BE: Small-cell lung cancer. Lancet 366: 1385-1396, 2005.

3. Li C and Lu H: Adenosquamous carcinoma of the lung. OncoTargets Ther 11: 4829-4835, 2018

4. Scheff RJ and Schneider BJ: Non-small-cell lung cancer: treatment of late stage disease: chemotherapeutics and new frontiers. Semin Intervent Radiol 30: 191-198, 2013.

5. Yu HA, Paz-Ares LG, Yang JC, Lee KH, Garrido P, Park K, Kim JH, Lee DH, Mao H, Wijayawardana SR, et al: Phase I study of the efficacy and safety of ramucirumab in combination with osimertinib in advanced T790M-positive EGFR-mutant non-small cell lung cancer. Clin Cancer Res 27: 992-1002, 2021.

6. Liu Y, Gao GF, Minna JD, Williams NS and Westover KD: Loss of wild type KRAS in KRASMUT lung adenocarcinoma is associated with cancer mortality and confers sensitivity to FASN inhibitors. Lung Cancer 153: 73-80, 2021.

7. Denisenko TV, Budkevich IN and Zhivotovsky B: Cell death-based treatment of lung adenocarcinoma. Cell Death Dis 9: 117, 2018.

8. Rutkowski MJ, Sughrue ME, Kane AJ, Mills SA and Parsa AT: Cancer and the complement cascade. Mol Cancer Res 8: $1453-1465,2010$.

9. Gros P, Milder FJ and Janssen BJ: Complement driven by conformational changes. Nat Rev Immunol 8: 48-58, 2008.

10. Kleczko EK, Kwak JW, Schenk EL and Nemenoff RA: Targeting the complement pathway as a therapeutic strategy in lung cancer. Front Immunol 10: 954, 2019.

11. Walport MJ: Complement. First of two parts. N Engl J Med 344: 1058-1066, 2001

12. Walport MJ: Complement. Second of two parts. N Engl J Med 344: 1140-1144, 2001.

13. Lee MJ, Na K, Jeong SK, Lim JS, Kim SA, Lee MJ, Song SY, Kim H, Hancock WS and Paik YK: Identification of human complement factor B as a novel biomarker candidate for pancreatic ductal adenocarcinoma. J Proteome Res 13: 4878-4888, 2014.

14. Low HP, Tiwari A, Janjanam J, Qiu L, Chang CI, Strohsnitter WC, Norwitz ER, Tam SW, Evans JE, Green KM, et al: Screening preeclamptic cord plasma for proteins associated with decreased breast cancer susceptibility. Genomics Proteomics Bioinformatics 11: 335-344, 2013.

15. Byrne SN, Hammond KJ, Chan CY, Rogers LJ, Beaugie C, Rana S, Marsh-Wakefield F, Thurman JM and Halliday GM: The alternative complement component factor B regulates UV-induced oedema, systemic suppression of contact and delayed hypersensitivity, and mast cell infiltration into the skin. Photochem Photobiol Sci 14: 801-806, 2015.

16. Andoh A, Fujiyama Y, Sakumoto H, Uchihara H, Kimura T, Koyama $\mathrm{S}$ and Bamba T: Detection of complement C3 and factor B gene expression in normal colorectal mucosa, adenomas and carcinomas. Clin Exp Immunol 111: 477-483, 1998.

17. Suman S, Basak T, Gupta P, Mishra S, Kumar V, Sengupta S and Shukla Y: Corrigendum to 'Quantitative proteomics revealed novel proteins associated with molecular subtypes of breast cancer' [Journal of Proteomics 148, (2016) 183-193]. J Proteomics 208: 103507, 2019.

18. Kwak JW, Laskowski J, Li HY, McSharry MV, Sippel TR, Bullock BL, Johnson AM, Poczobutt JM, Neuwelt AJ, Malkoski SP, et al: Complement Activation via a C3a Receptor Pathway Alters CD4 ${ }^{+}$T Lymphocytes and Mediates Lung Cancer Progression. Cancer Res 78: 143-156, 2018.

19. Azam F, Latif MF, Farooq A, Tirmazy SH, AlShahrani S, Bashir S and Bukhari N: Performance status assessment by using ECOG (Eastern Cooperative Oncology Group) score for cancer patients by oncology healthcare professionals. Case Rep Oncol 12: 728-736, 2019.
20. Barbetta C, Allgar V, Maddocks M, Ribeiro C, Wilcock A, Currow DC, Phillips J and Johnson MJ: Australia-modified Karnofsky Performance Scale and physical activity in COPD and lung cancer: an exploratory pooled data analysis. BMJ Support Palliat Care: Jun 11, 2019 (Epub ahead of print). doi: 10.1136/ bmjspcare-2019-001869.

21. Woodard GA, Jones KD and Jablons DM: Lung Cancer Staging and Prognosis. Cancer Treat Res 170: 47-75, 2016.

22. Wishart DS: Emerging applications of metabolomics in drug discovery and precision medicine. Nat Rev Drug Discov 15: 473-484, 2016

23. Livak KJ and Schmittgen TD: Analysis of relative gene expression data using real-time quantitative PCR and the $2(-\Delta \Delta \mathrm{C}(\mathrm{T}))$ method. Methods 25: 402-408, 2001.

24. Gold B, Merriam JE, Zernant J, Hancox LS, Taiber AJ, Gehrs K, Cramer K, Neel J, Bergeron J, Barile GR, et al; AMD Genetics Clinical Study Group: Variation in factor B (BF) and complement component $2(\mathrm{C} 2)$ genes is associated with age-related macular degeneration. Nat Genet 38: 458-462, 2006.

25. Colten HR: Biosynthesis of the MHC-linked complement proteins $(\mathrm{C} 2, \mathrm{C} 4$ and factor $\mathrm{B})$ by mononuclear phagocytes. Mol Immunol 19: 1279-1285, 1982.

26. Canavese F, Botnari A, Andreacchio A, Marengo L, Samba A Dimeglio A, Pereira B, Mansour M and Rousset M: Displaced tibial shaft fractures with intact fibula in children: Nonoperative management versus operative treatment with elastic stable intramedullary nailing. J Pediatr Orthop 36: 667-672, 2016.

27. Chen M, Muckersie E, Robertson M, Forrester JV and Xu H Up-regulation of complement factor $\mathrm{B}$ in retinal pigment epithelial cells is accompanied by complement activation in the aged retina. Exp Eye Res 87: 543-550, 2008.

28. Ostvik AE, Granlund A, Gustafsson BI, Torp SH, Espevik T, Mollnes TE, Damås JK and Sandvik AK: Mucosal toll-like receptor 3-dependent synthesis of complement factor B and systemic complement activation in inflammatory bowel disease. Inflamm Bowel Dis 20: 995-1003, 2014.

29. Potter BJ, Brown DJ, Watson A and Jewell DP: Complement inhibitors and immunoconglutinins in ulcerative colitis and Crohn's disease. Gut 21: 1030-1034, 1980.

30. Jiang DK, Ma XP, Yu H, Cao G, Ding DL, Chen H, Huang HX, Gao YZ, Wu XP, Long XD, et al: Genetic variants in five novel loci including CFB and CD40 predispose to chronic hepatitis B. Hepatology 62: 118-128, 2015.

31. Ciriello G, Gatza ML, Beck AH, Wilkerson MD, Rhie SK, Pastore A, Zhang H, McLellan M, Yau C, Kandoth C, et al; TCGA Research Network: Comprehensive molecular portraits of invasive lobular breast cancer. Cell 163: 506-519, 2015.

32. Kim SH, Lee MJ, Hwang HK, Lee SH, Kim H, Paik YK and Kang CM: Prognostic potential of the preoperative plasma complement factor B in resected pancreatic cancer: A pilot study. Cancer Biomark 24: 335-342, 2019.

33. Lim LY, Tan GH, Zainuddin ZM, Fam XI, Goh EH, Syaris OS, Yahaya A and Singam P: Prospective evaluation of using multiparametric magnetic resonance imaging in cognitive fusion prostate biopsy compared to the standard systematic 12-core biopsy in the detection of prostate cancer. Urol Ann 12: 276-282, 2020.

34. Bauer D, Mazzio E, Hilliard A, Oriaku ET and Soliman KF: Effect of apigenin on whole transcriptome profile of TNF $\alpha$-activated MDA-MB-468 triple negative breast cancer cells. Oncol Lett 19: 2123-2132, 2020

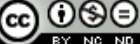

This work is licensed under a Creative Commons Attribution-NonCommercial-NoDerivatives 4.0 International (CC BY-NC-ND 4.0) License. 